Article

\title{
Comparative Study on the Effect of Protonation Control for Resistive Gas Sensor Based on Close-Packed Polypyrrole Nanoparticles
}

\author{
Wooyoung Kim ${ }^{1}$, Sunghun Cho ${ }^{2, *}$ and Jun Seop Lee ${ }^{3, *(1)}$ \\ 1 School of Chemical and Biological Engineering, Seoul National University, 1 Gwanak-ro, Gwanak-gu, \\ Seoul 151-742, Korea; kimgregorio@snu.ac.kr \\ 2 School of Chemical Engineering, Yeungnam University, Gyeongsan 38541, Korea \\ 3 Department of Materials Science and Engineering, Gachon University, 1342 Seongnam-Daero, Sujeong-Gu, \\ Seongnam-Si, Gyeonggi-Do 13120, Korea \\ * Correspondence: shcho83@ynu.ac.kr (S.C.); junseop@gachon.ac.kr (J.S.L.); Tel.: +82-31-750-5814 (S.C.); \\ +82-53-810-2535 (J.S.L.)
}

Received: 23 January 2020; Accepted: 3 March 2020; Published: 8 March 2020

\begin{abstract}
Conducting polymers are often used as sensor electrodes due to their conjugated chain structure, which leads to high sensitivity and rapid response at room temperature. Numerous studies have been conducted on the structures of conducting polymer nanomaterials to increase the active surface area for the target materials. However, studies on the control of the chemical state of conducting polymer chains and the modification of the sensing signal transfer with these changes have not been reported. In this work, polypyrrole nanoparticles (PPyNPs), where is PPy is a conducting polymer, are applied as a sensor transducer to analyze the chemical sensing ability of the electrode. In particular, the protonation of PPy is adjusted by chemical methods to modify the transfer sensing signals with changes in the polymer chain structure. The PPyNPs that were modified at $\mathrm{pH} 1$ exhibit high sensitivity to the target analyte (down to $1 \mathrm{ppb}$ of $\mathrm{NH}_{3}$ ) with short response and recovery times of less than $20 \mathrm{~s}$ and $50 \mathrm{~s}$, respectively, at $25^{\circ} \mathrm{C}$.
\end{abstract}

Keywords: polypyrrole; protonation; chemical sensor; nanoparticles; chemical state; ammonia gas

\section{Introduction}

In the past few decades, chemical gas sensors have been widely used in several applications, including the detection of combustible, flammable, and toxic gases [1-5]. Various semiconductive materials are used as transducers for chemical sensors, and extensive investigations have been conducted to improve their performance [6-9]. The critical indicators for sensor performance include high sensitivity to the target analyte, short response and recovery times, and cycle stability $[10,11]$. Among these, the sensitivity and cycle stability are optimized via the control of the morphology of electrode materials. However, the response and recovery times can be adjusted by modifying the semiconductive properties of the electrode material, unlike other factors.

Conducting polymers, which are semiconductive materials, have been investigated for sensor applications, owing to their versatility originating from the conjugated backbone structure consisting of alternating single and double bonds [12,13]. Conducting polymers have been used as sensor transducers with inherent electronic, optical, and mechanical transducing properties because of their unique properties [14-16]. Considerable efforts have been directed toward the fabrication of nanometer-scale conducting polymers, owing to their beneficial characteristics, such as small size, high surface-to-volume ratio, and amplified signals, to enhance the sensitivity [17-20]. In recent years, several studies have focused on increasing the surface area of conducting polymer nanomaterials 
to maximize the interactions between the transducers and analytes [21-24]. Cho et al. fabricated multidimensional conductive nanofilms that consisted of graphene-coated carboxyl polypyrrole nanowires for application as a Hepatitis B surface antigen aptamer sensor electrode [21]. Bao et al. synthesized three-dimensional polyaniline hydrogels with high surface areas and conductivities for application in glucose oxidase sensors [24]. However, research on controlling the electrical signal transmission speed of the electrode materials is limited.

Among the most popular conducting polymers, polypyrrole (PPy) exhibits several beneficial characteristics, including high electrical conductivity, redox property, and environmental stability [25-27]. PPy has been extensively investigated in sensor applications, owing to its unique chemical and electrical properties [28-30]. In particular, PPy has different electronic band structures, such as neutral, polaron, and bipolaron states, which can be facilely modified by controlling the doping ratio [31,32]. However, its structure rather than the doping ratio is more often modified for application in sensor transducer materials.

Herein, the sensing performance of polypyrrole nanoparticles (PPyNPs) as a sensor transducer in different chemical states is investigated. First, PPyNPs with a uniform diameter of $60 \mathrm{~nm}$ were synthesized via a modified monodisperse method, and then subjected to chemical treatment while using aqueous solutions of different $\mathrm{pH}$ levels ( $\mathrm{pH}$ 1-13). The charge carrier (hole) density and diameter of the particles decreased with an increase in $\mathrm{pH}$, owing to the deprotonation of the polymer chain under basic conditions. The nanoparticles with controlled protonation were subsequently introduced on an interdigitated array electrode surface via spin-coating for application as an $\mathrm{NH}_{3}$ gas-sensing transducer. The sensor electrode at $\mathrm{pH} 1$ exhibited high sensitivity to the target analyte (down to $1 \mathrm{ppb}$ of $\mathrm{NH}_{3}$ ), with short response and recovery times of less than $20 \mathrm{~s}$ and $50 \mathrm{~s}$, respectively, at $25^{\circ} \mathrm{C}$.

\section{Materials and Methods}

\subsection{Materials}

Iron chloride (III) $\mathrm{FeCl}_{3}$ (97\%), poly(vinyl alcohol) (PVA, Mw 9000), dodecyl trimethylammonium bromide (DTAB, $\geq 98 \%$ ), and pyrrole (98\%) were purchased from Sigma-Aldrich and used as purchased. Sodium hydroxide $(\mathrm{NaOH}, 95 \%)$ and hydrochloric acid $(\mathrm{HCl}, 35 \sim 37 \%)$ were purchased from Samchun Pure Chemical Company (Pyeongtaek-si, Korea).

\subsection{Fabrication of PPyNPs}

PPyNPs were prepared while using PVA, $\mathrm{FeCl}_{3}$, and pyrrole in distilled water. A $0.5 \mathrm{~g}$ sample of PVA in distilled water $(50 \mathrm{~mL})$ was magnetically stirred $(300 \mathrm{rmp})$ at $25^{\circ} \mathrm{C}$ for $1 \mathrm{~h}$. After the dissolution of PVA in distilled water, $0.02 \mathrm{~mol}$ of $\mathrm{FeCl}_{3}$ was added into the aqueous PVA solution with $300 \mathrm{rpm}$. After $1 \mathrm{~h}$ of equilibration, $0.06 \mathrm{~mol}$ of pyrrole monomer was rapidly added into the aqueous $\mathrm{PVA} / \mathrm{FeCl}_{3}$ solution and then stirred for $4 \mathrm{~h}$. After the completion of polymerization, the resulting nanoparticles were separated from the dispersion by centrifugation and then washed several times with water to remove the impurities. The remaining PPyNP precipitate was dried in a vacuum oven at room temperature for $6 \mathrm{~h}$.

\subsection{Protonation Control of PPyNPs}

PPyNPs were added to the aqueous solutions with different $\mathrm{pH}$ values and sonicated for $1 \mathrm{~h}$ to uniformly disperse the nanoparticles in the solution. Except for $\mathrm{pH} 7, \mathrm{HCl}$ and $\mathrm{NaOH}$ were used for realizing acidic and basic conditions, respectively. The $\mathrm{pH} 7$ solution is used commercial phosphate buffers (Samchun Co.). Subsequently, the solutions with PPyNPs were stirred at a low rate for an additional $1 \mathrm{~h}$ to allow for the completion of the reaction between the particles and solvent. The mixed solution was centrifuged and dried overnight at $60^{\circ} \mathrm{C}$ in an oven. 


\subsection{Electrical Sensing Measurement of Hazardous Gases}

Spin-coating was conducted to obtain a uniformly coated sensor electrode array. In detail, $0.1 \mathrm{wt} \%$ PPyNP containing aqueous solution is used. $10 \mu \mathrm{L}$ of the solution was deposited on the electrode and following spin-coating was conducted $(1000 \mathrm{rpm}, 60 \mathrm{~s})$. Afterwards, the electrode was dried at $60^{\circ} \mathrm{C}$ in an inert atmosphere for $6 \mathrm{~h}$ to obtain good electrical ohmic contact between the PPyNPs and electrodes. The sensor electrode was placed inside the vacuum chamber $\left(10^{0} \mathrm{Torr}\right)$ and connected to a source meter to monitor the resistance change while using a computer. In this experiment, a bubbler was used to vaporize the organic compounds from ammonia and methanol solutions and to mix the vapors with $\mathrm{N}_{2}$ gas using a mass flow controller to modify the concentrations of the analyte gases. The sensor electrode was exposed to various concentrations of analyte gases (1 ppb-100 ppm) for $100 \mathrm{~s}$ and then purged with $\mathrm{N}_{2}$ gas to recover the conductance. The measurement was performed by applying a constant current of $10^{-6} \mathrm{~A}$ to the electrode and the sensitivity was calculated by measuring the normalized electrical resistance change, $\Delta R / R_{0}=\left(R-R_{0}\right) / R_{0}$, where $R$ and $R_{0}$ are the measured real-time resistance and initial resistance, respectively. The response time was defined as the time that is required for a sensor to attain $90 \%$ of the maximum signal after gas exposure, and the recovery time was defined as the time that is required by a sensor to attain $10 \%$ of the preceding signal after purging with $\mathrm{N}_{2}$ gas. The sensing performance was evaluated under ambient conditions (ca. 20\% relative humidity).

\subsection{Characterization}

The field emission-scanning electron microscopy (FE-SEM) images were obtained while using the JSM-6701F system (JEOL Ltd., Tokyo, Japan). X-ray photoelectron spectroscopy (XPS) data were acquired using a Sigma probe (Thermo, Waltham, MA, USA). The electrical conductivity was measured using a Keithley 2400 system. Raman spectra were obtained while using a LabRam Aramis (Horiba Jobin Yvon, Bensheim, Germany) spectrometer. Ultra-violet visible and near infra-red (UV-vis-NIR) spectra were collected on a UV-3600 Plus spectrophotometer (Shimadzu, Tokyo, Japan).

\section{Results and Discussion}

\subsection{Transformation of Polymer Chain Structure}

Uniform PPyNPs (ca. diameter of $60 \mathrm{~nm}$ ) as a sensor transducer material were synthesized via a modified monodisperse method [30]. Here, polyvinyl alcohol (PVA) and ferric chloride $\left(\mathrm{FeCl}_{3}\right)$ were dissolved in deionized water to form the PVA/iron cation $\left(\mathrm{Fe}^{3+}\right)$ complex that acted as the oxidizing and forming agent during the polymerization reaction. Subsequently, the pyrrole monomer was introduced into the aqueous $\mathrm{PVA} / \mathrm{Fe}^{3+}$ complex solution with vigorous stirring to allow for the formation of PPyNPs at the reactive sites, where the pyrrole monomer contacted the PVA/ $\mathrm{Fe}^{3+}$ complex.

The charge carrier density and mobility of the PPy chain change reversibly with different protonation states, as shown in Figure 1. The expanded state of the polymer chain is formed upon protonation, while the contracted state is formed upon deprotonation. The PPyNPs were dispersed in aqueous solutions with $\mathrm{pH}$ values ranging from $\mathrm{pH} 1$ to 13 to achieve different chemical states of the polymer chains. The concentrations of the charge carriers in the polymer chain were determined while using UV-vis-NIR spectroscopy. UV-vis-NIR spectra were collected to examine the types and transition of the polymer chain at various $\mathrm{pH}$ values (Figure 2). The polymer nanoparticles at $\mathrm{pH} 1$ show the highest absorption intensity of the band above $1000 \mathrm{~nm}$ and around the $450 \mathrm{~nm}$ region because of the high carrier density in the polymer chain, which causes a reduction in the band gap, as shown in Figure 2a. An increase in $\mathrm{pH}$ results in the reduction of the band intensity above $1000 \mathrm{~nm}$ and a blue shift of the medium intensity around $450 \mathrm{~nm}$. These spectral changes show that a high $\mathrm{pH}$ results in the loss of protons (reduction) from the polymer chain [33]. Thus, the polymer chains transform from expanded to contracted states with an increase in $\mathrm{pH}$. Figure $2 \mathrm{~b}$ also shows the maximum absorption at different wavelengths $(300,450$, and $1000 \mathrm{~nm}$ ). The absorption of the bands in the high-NIR region $(1000 \mathrm{~nm})$ continuously decreases as the chain structure changes from an expanded to contracted state. 
In addition, the intensity of absorption at $450 \mathrm{~nm}$ (visible) shifts to a low wavelength with an increase in intensity at $300 \mathrm{~nm}$ (UV) due to the same reason.<smiles>CC(C)(C)c1ccc(-c2ccc(C3=NC(=C4C=CC(c5ccc(C(F)(F)F)[nH]5)=N4)C=C3)[nH]2)[nH]1</smiles>

Contracted state (reduced form)

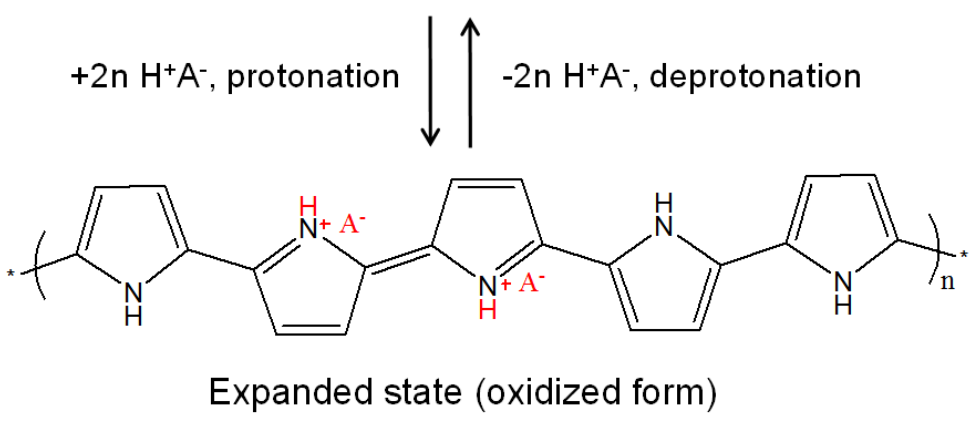

Figure 1. Chemical structures of polypyrrole (PPy) in different chemical states.
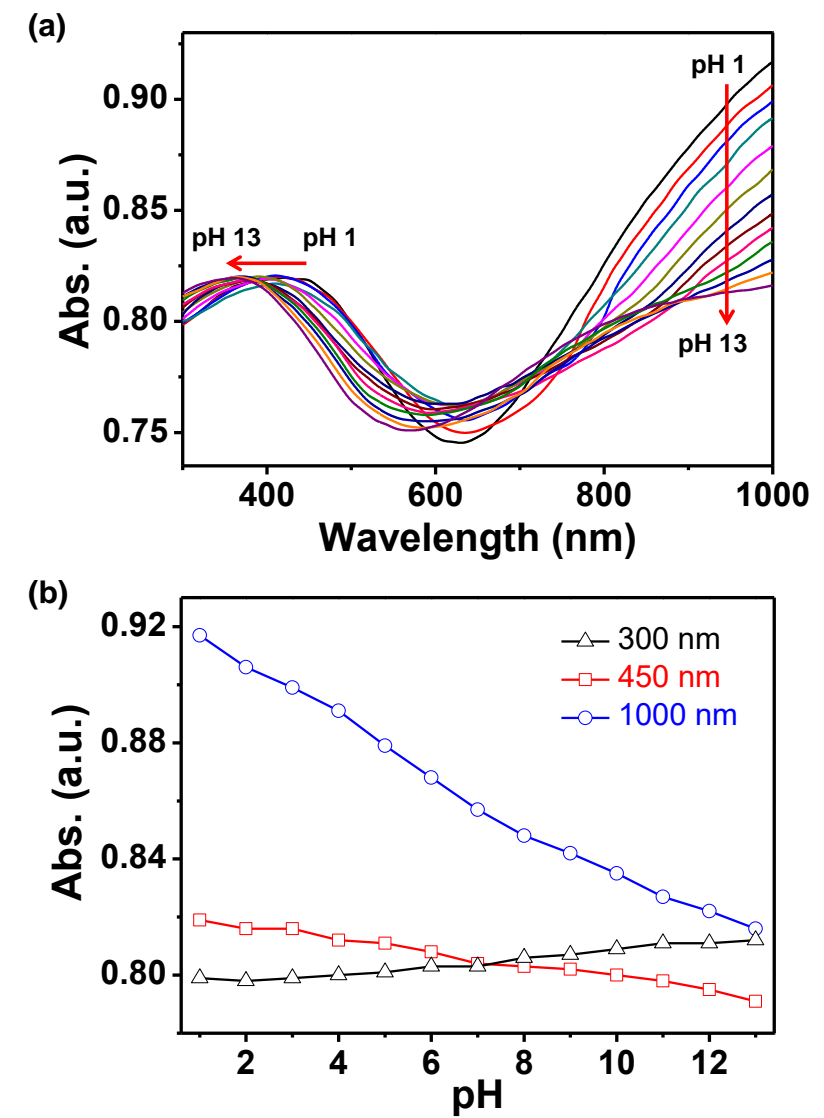

Figure 2. (a) Ultra-violet visible and near infra-red (UV-vis-NIR) absorption spectra of polypyrrole nanoparticles (PPyNPs) at different $\mathrm{pH}$ values. (b) Normalized absorption spectra of PPyNPs with a change in $\mathrm{pH}$ at different wavelengths (black for $300 \mathrm{~nm}$, red for $450 \mathrm{~nm}$, and blue for $1000 \mathrm{~nm}$ ).

Raman spectroscopy was performed to characterize the different chemical states of the PPyNPs. Figure 3a shows the Raman spectra at different $\mathrm{pH}$ values ( $\mathrm{pH} 1-13)$. The bands at 1049 and $1332 \mathrm{~cm}^{-1}$ corresponded to the in-plane $\mathrm{C}-\mathrm{H}$ and $\mathrm{C}-\mathrm{C}$ stretching vibrations, respectively. Moreover, the strong 
bands at 1421 and $1591 \mathrm{~cm}^{-1}$ are attributed to the $C=C$ symmetric and $C=C$ asymmetric vibrations, respectively. These bands shift to 1415 and $1562 \mathrm{~cm}^{-1}$, respectively, with an increase in $\mathrm{pH}$ indicating the transformation of the polymer chains from quinoid to benzenoid structures. Specifically, the positively doped species are located at the geometrical defects and form the quinoid structure. Therefore, high $\mathrm{pH}$ decreases the amount of (bi)polarons and changes the polymer chain structures to quinoid structures [34, 35].

(a)

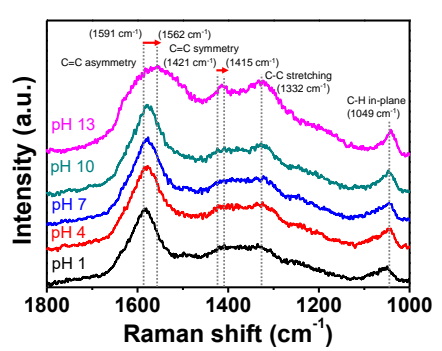

(d)

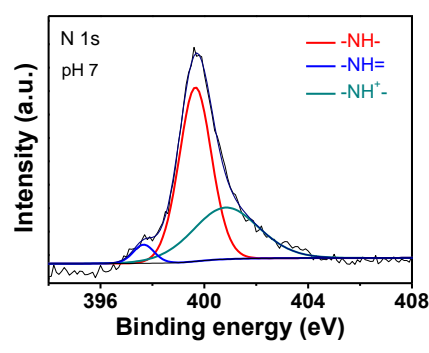

(b)

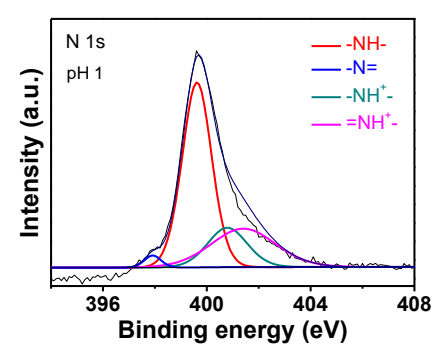

(e)

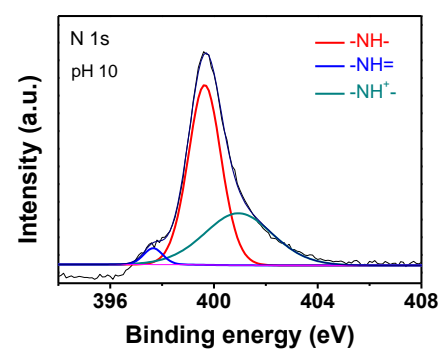

(c)
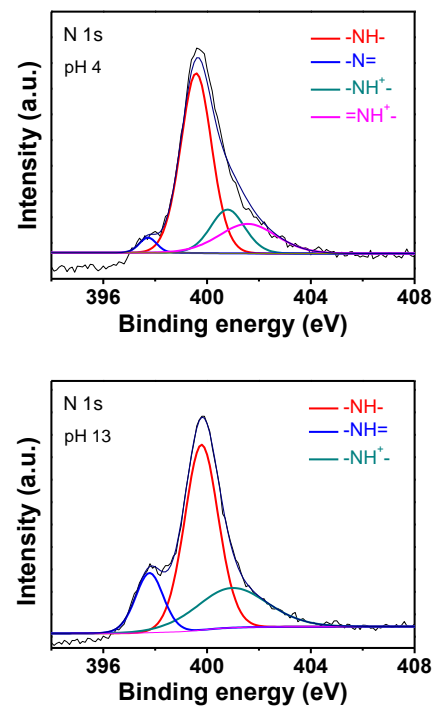

Figure 3. (a) Raman spectra of PPyNPs at different $\mathrm{pH}$ values (black: $\mathrm{pH} 1$, red: $\mathrm{pH}$ 4, blue: $\mathrm{pH} 7$, green: $\mathrm{pH}$ 10, and pink: $\mathrm{pH}$ 13). N 1s X-ray photoelectron spectra (XPS) of PPyNPs at different $\mathrm{pH}$ values: (b) pH 1, (c) pH 4, (d) pH 7, (e) $\mathrm{pH}$ 10, and (f) $\mathrm{pH} 13$.

Furthermore, high-resolution N 1s X-ray photoelectron spectroscopy (XPS) was employed to confirm the change in the chemical state of the PPy chain at different $\mathrm{pH}$ values (Figure $3 \mathrm{~b}-\mathrm{f}$ ). The peaks at ca. $400 \mathrm{eV}$ (related to the neutral amine $\mathrm{N}$ of the pyrrole unit (-NH-)) and ca. $398 \mathrm{eV}$ (attributed to imine $(-\mathrm{N}=))$ are observed in all cases [36]. For the nanoparticles at $\mathrm{pH} 1$ and 4 , two types of positive nitrogen peaks, including the $\mathrm{N}$ peaks at $403.0 \mathrm{eV}\left(=\mathrm{NH}^{+}-\right)$and $401.4 \mathrm{eV}\left(-\mathrm{NH}^{+}-\right)$, are observed. At high $\mathrm{pH}$, a peak corresponding to the positively charged $\mathrm{N}\left(-\mathrm{NH}^{+}-\right)$is observed at $401.4 \mathrm{eV}$. While using these data, the protonation of the nanoparticles can be calculated in terms of the ratio of the positive nitrogen peak area over the total nitrogen peak area $\left(\mathrm{N}^{+} / \mathrm{N}_{\text {total }}\right)$ [37]. On treatment with a base, $\mathrm{N}^{+} / \mathrm{N}_{\text {total }}$ is lower than those that were obtained under other conditions, because the positive nitrogen $\left(\mathrm{N}^{+}\right)$content in the structure decreases (i.e., deprotonation) at high $\mathrm{pH}$. Thus, an increase in the protonation (i.e., lower $\mathrm{pH}$ ) of the particle increases the amount of positively charged $\mathrm{N}$ in the structure.

Field emission scanning electron microscopy (FE-SEM) measurements were performed for each nanoparticle to determine the morphological transformations of the PPyNPs with varying $\mathrm{pH}$ values (Figure 4). The morphologies of the PPyNPs change reversibly at different $\mathrm{pH}$ values through the osmotic effect of the counter ion $\left(\mathrm{Cl}^{-}\right)$. Upon protonation, the electrons are ejected from the PPyNPs and a positive charge develops in the polymer chain. Thereafter, the anions in the solution can displace and induce a volume expansion in the polymer through the insertion of the counter ions and subsequent uptake of the solvent. Consequently, the average diameter of the individual nanoparticles reduces from $68.71 \mathrm{~nm}$ at $\mathrm{pH} 1$ to $60.63 \mathrm{~nm}$ at $\mathrm{pH} 13$. 


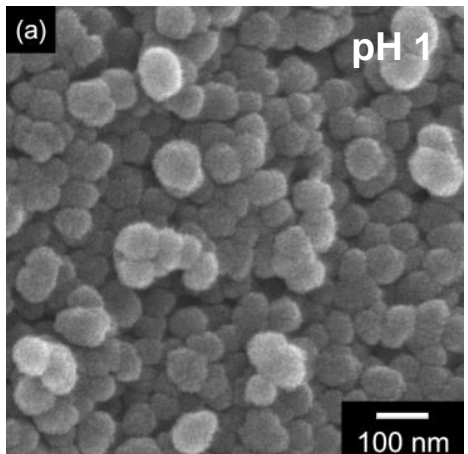

(d)
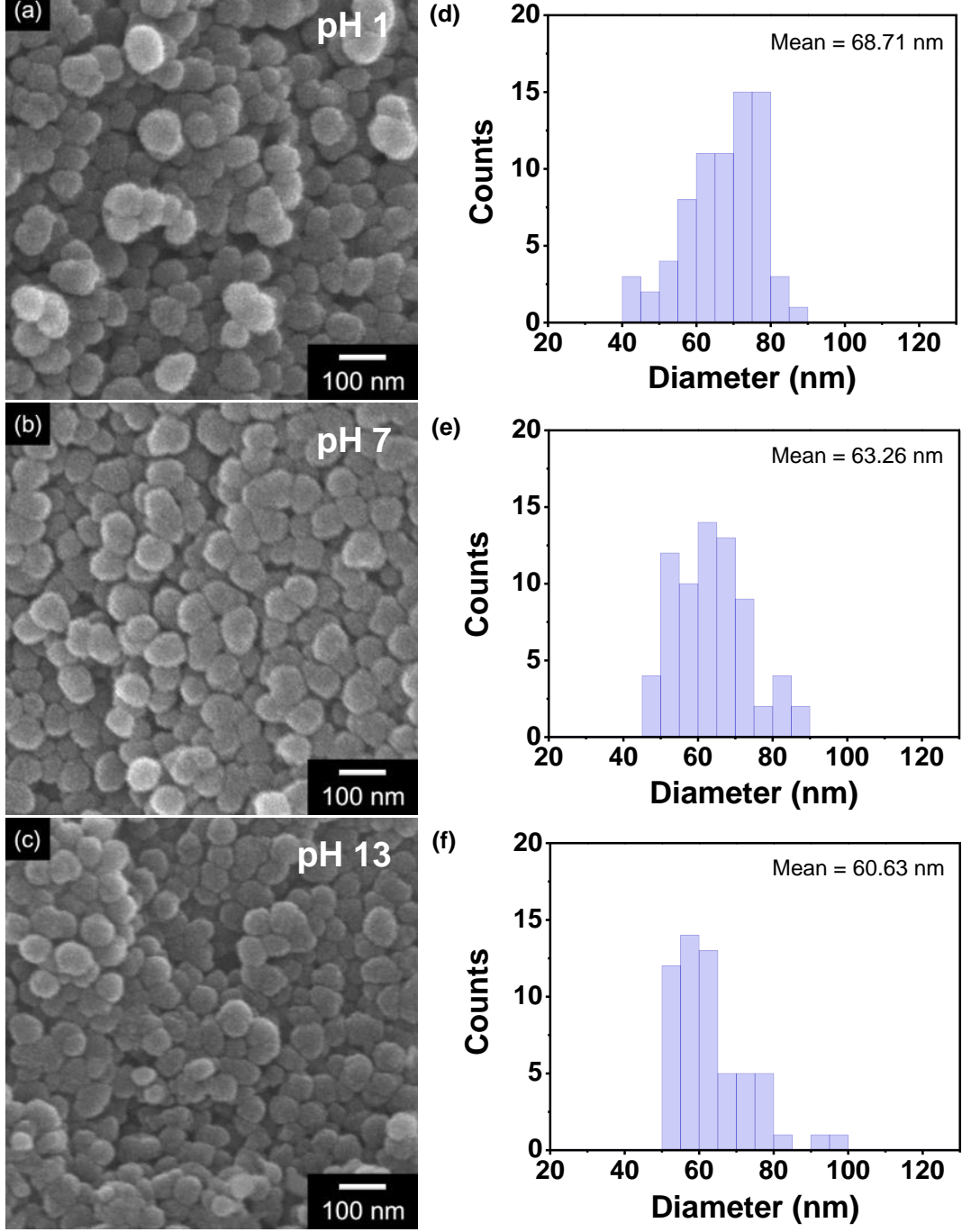

Figure 4. $(\mathbf{a}-\mathbf{c})$ Field emission scanning electron micrographs of PPyNPs and $(\mathbf{d}-\mathbf{f})$ size distribution of the nanoparticles within a unit area $(1 \mu \mathrm{m} \times 1 \mu \mathrm{m})$ at different $\mathrm{pH}$ values $((\mathbf{a}, \mathbf{d}): \mathrm{pH} 1 ;(\mathbf{b}, \mathbf{e}): \mathrm{pH} 7 ;(\mathbf{c}, \mathbf{f})$ : $\mathrm{pH}$ 13).

\subsection{Electrical Conductivity}

The electrical conductivity of the PPyNPs is investigated to determine the charge transfer with changes in $\mathrm{pH}$ (Figure 5). Different protonation levels control the electrical conductivity $\left(128 \mathrm{~S} \mathrm{~cm}^{-1}\right.$ at $\mathrm{pH} 1$ to $1.9 \mathrm{~S} \mathrm{~cm}^{-1}$ at $\mathrm{pH} 13$ ), as an increase in acidity increases the carrier density (hole) in polymer chains. This is because proton doping occurs during the acidic treatment and the $\beta$-carbon in the polymer chain structure is preferentially protonated [38]. Protonation is essential for inducing the short-range ordering of the polymer backbone and for the efficient proton transport in the conjugated chains, and it is achieved by increasing the amount of $C=C$ stretching [39]. Additionally, a significant decrease in the electrical conductivity is observed under basic conditions due to a drastic reduction in the number of charge carriers during deprotonation. 


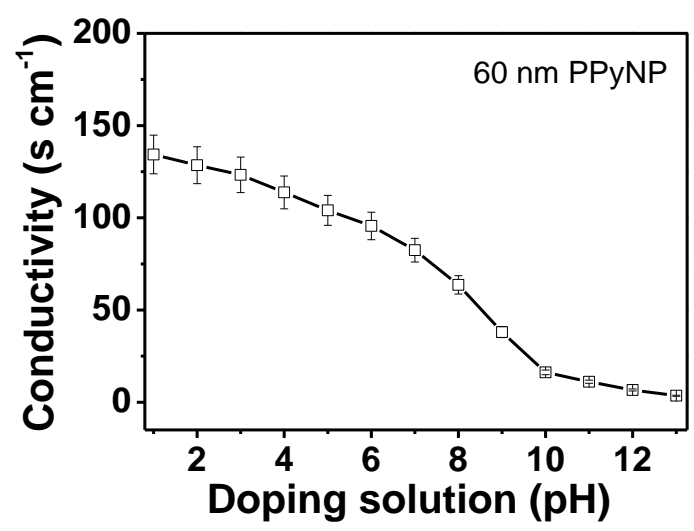

Figure 5. Electrical conductivities of PPyNPs at various $\mathrm{pH}$ values.

\subsection{Preparation of PPyNP-Based Sensor Electrode}

PPyNPs were immobilized on the interdigitated array electrode to determine the sensing performance of the particles as a function of $\mathrm{pH}$. The conduction pathway and effective surface area were utilized to compare the performance of the particles with the variation in their chemical state. Figure 6 shows the data for the source-drain current as a function of the source-drain voltage $\left(\mathrm{I}_{\mathrm{SD}}-\mathrm{V}_{\mathrm{SD}}\right)$ for the nanoparticles; the results indicate that the nanoparticles are in good electrical contact with the sensor substrate. However, the uniform decoration of the particles improves the conduction pathway, i.e., increases the sensitivity to the analyte. The randomly stacked arrangement of the PPyNPs that were prepared by drop-casting has a higher contact resistance than the uniform array that was obtained by spin-coating due to the interruption of the conduction pathway through the accumulated particle-particle assembly. Therefore, PPyNPs were deposited on the electrode via spin-coating to determine the sensing performance in real time with minimum contact resistance.
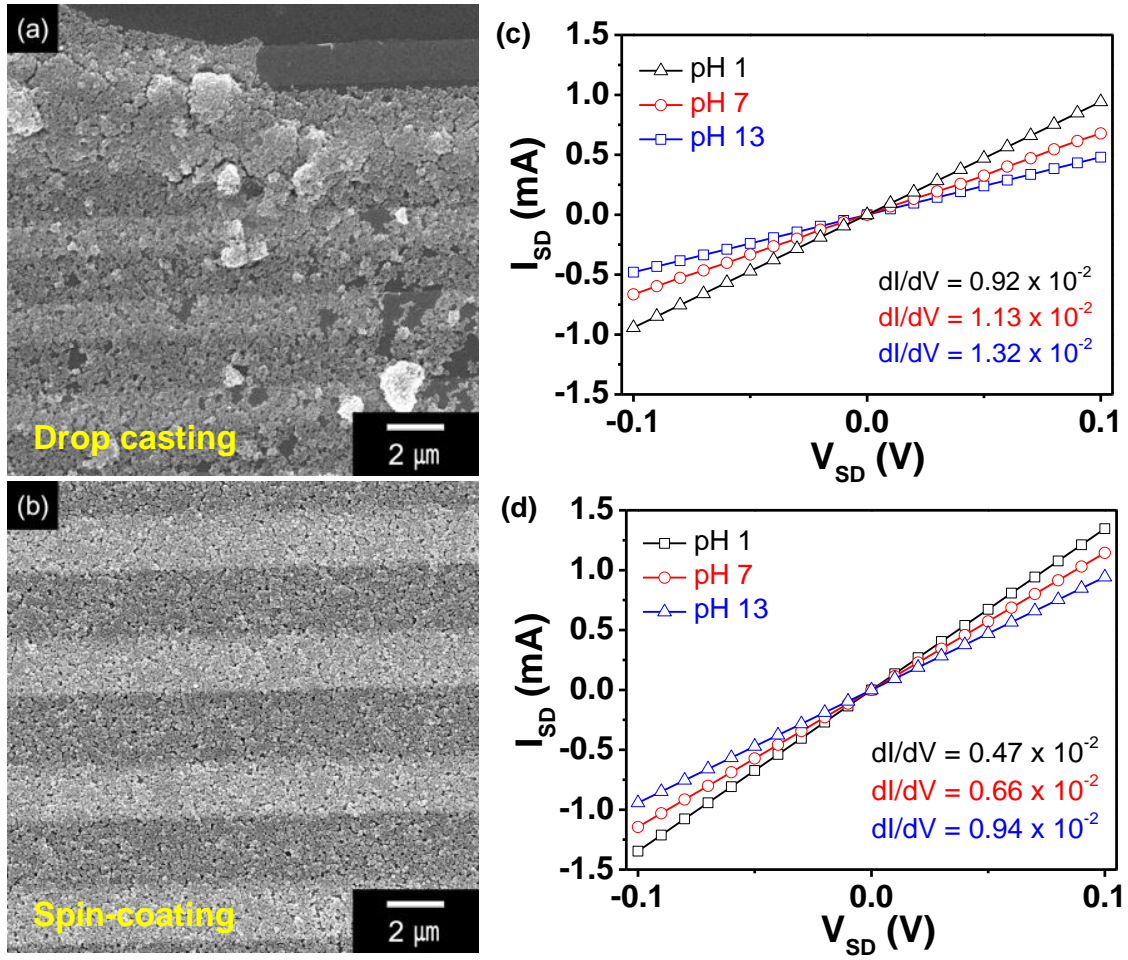

Figure 6. (a,b) Field emission scanning electron microscopy (FE-SEM) images and (c,d) source-drain voltage-current curves of the PPyNP-decorated sensor electrodes prepared by $(\mathbf{a}, \mathbf{c})$ drop-casting or $(\mathbf{b}, \mathbf{d})$ spin-coating. 


\subsection{Comparison of Sensing Performance}

The changes in the real-time responsive resistance were measured for different concentrations of gases at $25{ }^{\circ} \mathrm{C}$. Figure 7 a shows the sensing performance of the electrodes toward $\mathrm{NH}_{3}$ gas, which is one of the reducing gases, with respect to the $\mathrm{pH}$. The $\mathrm{NH}_{3}$ molecules generate electrons, owing to the reduction characteristics during detection. These electrons are transferred to the PPyNPs, thereby reducing the amount of the charge carriers (hole) in the polymer chain. Therefore, the resistance of the sensor electrode increases, owing to the p-type semiconductor characteristics of the polymer. The sensor electrodes exhibit being highly sensitive toward $\mathrm{NH}_{3}$ down to $10 \mathrm{ppb}$ due to the uniformly decorated nanoparticles on the IDA electrode. However, the response amounts of the electrodes display increase with reducing $\mathrm{pH}$ of solution. The more protonated PPyNPs (treated at $\mathrm{pH} 1$ ) have more holes in the chain structure, indicating a higher degree to change in external electron flow. On the other hand, the electrode that was treated at $\mathrm{pH} 13$ shows the smallest amount of resistance change, even though it has the smallest diameter. Thus, a reduction in the amount of holes, rather than a reduction in the surface area, has a greater effect on response change. Figure $7 \mathrm{~b}$ shows the recovery times of the electrodes, exhibiting a decrease from $59 \mathrm{~s}$ ( $\mathrm{pH}$ 13) to $19 \mathrm{~s}$ ( $\mathrm{pH}$ 1). The numerous charge carriers (hole) at $\mathrm{pH} 1$ induce the rapid transfer of electrons from the analyte and reduce the response time to $<20 \mathrm{~s}$. However, the recovery times of the electrodes exhibit similar values, i.e., $66 \mathrm{~s}$ for $\mathrm{pH} 13,61 \mathrm{~s}$ for $\mathrm{pH}$ 7 , and $49 \mathrm{~s}$ for $\mathrm{pH} \mathrm{1,} \mathrm{because} \mathrm{the} \mathrm{detachment} \mathrm{of} \mathrm{the} \mathrm{target} \mathrm{molecule} \mathrm{is} \mathrm{related} \mathrm{to} \mathrm{the} \mathrm{applied} \mathrm{external}$ energy rather than to the charge carriers and number of active sites (Figure 7c). Moreover, the sensing performance with decreasing $\mathrm{NH}_{3}$ concentration was also measured to confirm the reproducibility of the electrode. Figure $7 \mathrm{~d}$ shows that the sensing ability of the electrode remains constant, regardless of the direction of change in $\mathrm{NH}_{3}$ concentration. In addition, we also compared the response and recovery times of the electrode with the direction of concentration changes. The response time of the sensor electrode is similar (19 s) in both cases, as shown in Figure 7e. However, the recovery time for backward direction (60 s) presents a little longer than forward direction (49 s), due to exposure to large amount of $\mathrm{NH}_{3}$ molecules during detection (Figure 7f). Therefore, PPy-based sensor electrodes have good reproducibility for environmental changes.

Additionally, methanol $(\mathrm{MeOH})$ was used to determine the sensing ability for an oxidizing gas (opposite charge-transfer direction) (Figure 8a). The electrode oxidized at $\mathrm{pH} 1$ is superior to other electrodes for the same reason, as observed in the case of $\mathrm{NH}_{3}$. Figure $8 \mathrm{~b}$ shows the variation in the normalized resistance of the electrodes as a function of $\mathrm{NH}_{3}$ and $\mathrm{MeOH}$ gas concentrations. The linear-behavior range is larger for the electrode oxidized at $\mathrm{pH} 1\left(1 \mathrm{ppb}-10^{2} \mathrm{ppm}\right.$ for $\mathrm{NH}_{3}$ and $0.1-10^{3}$ ppm for $\mathrm{MeOH}$ ) than for the other electrodes. Therefore, the sensor electrode oxidized at $\mathrm{pH} 1$ can be effectively utilized as a signal transducer for detecting $\mathrm{NH}_{3}$ and $\mathrm{MeOH}$ gases at various concentrations. Additionally, outstanding cycle stability is required for use of the electrode materials in practical sensor devices. Figure $8 \mathrm{c}$ shows the change in the resistance of the electrode at $\mathrm{pH} 1$ upon periodic exposure

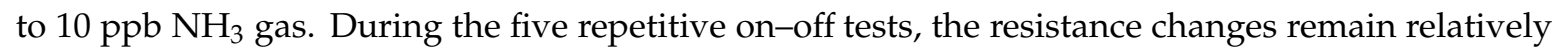
constant, without a delay in the response or recovery. Furthermore, the sensor electrodes maintain their sensing ability during exposure to $0.1 \mathrm{ppm}$ of $\mathrm{NH}_{3}$ over 15 days (Figure 9). 
(a)

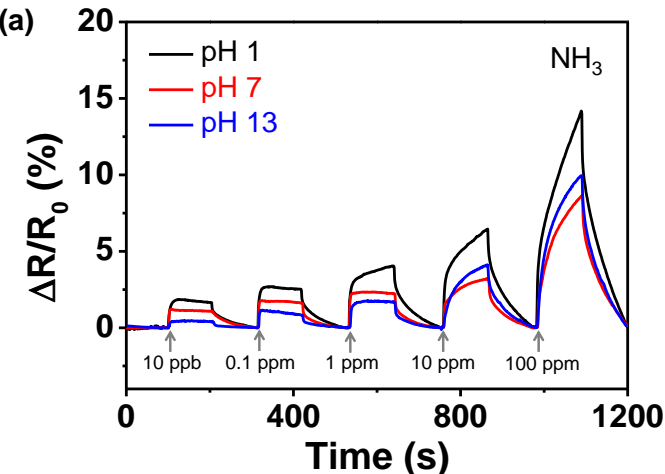

(c)

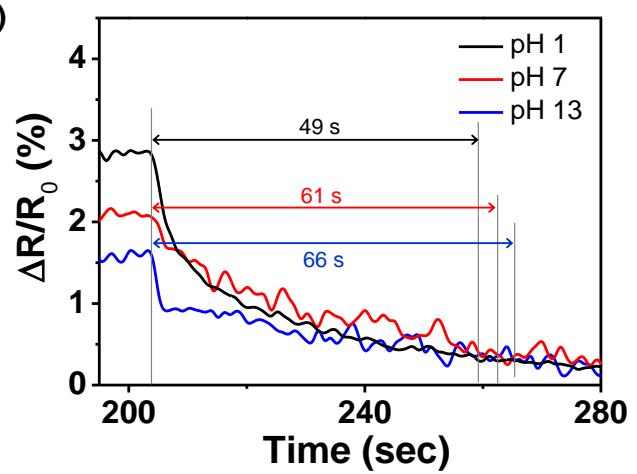

(e)

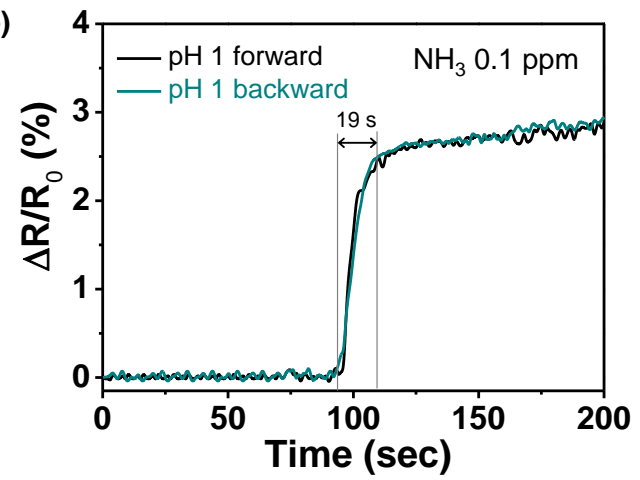

(b)

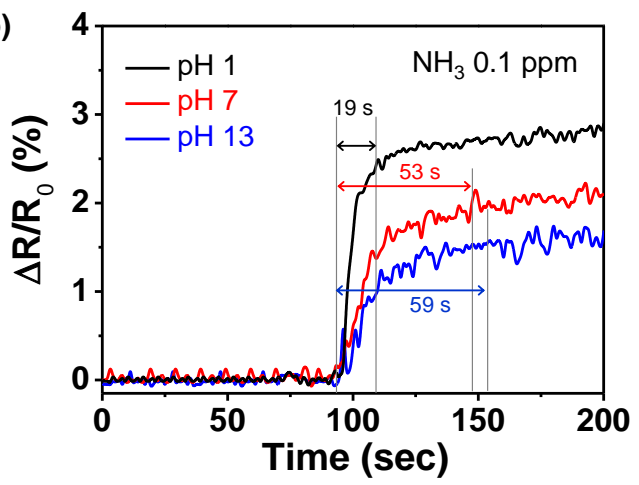

(d)

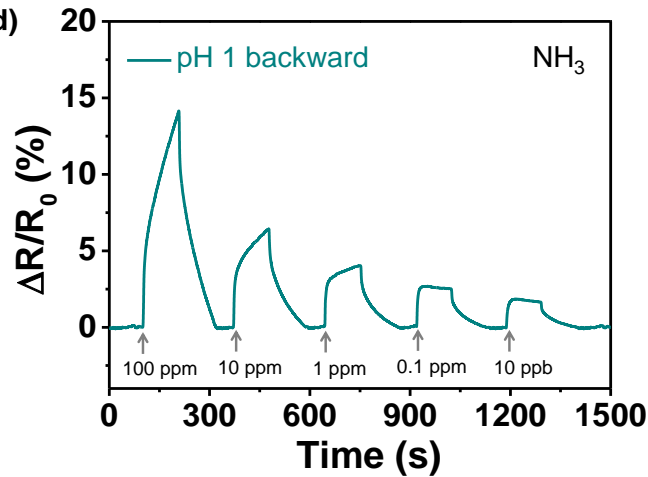

(f)

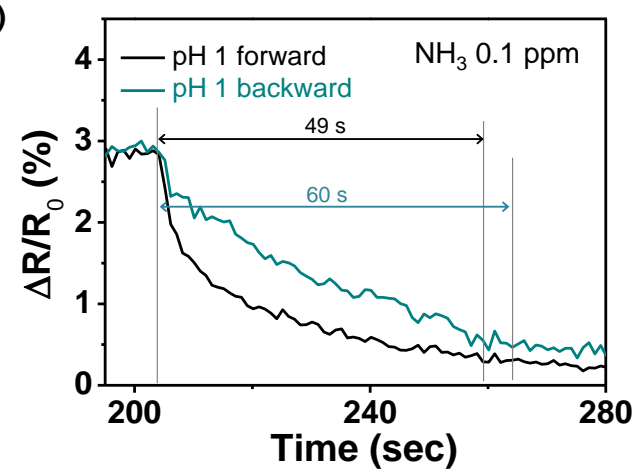

Figure 7. Reversible and reproducible responses are measured at a constant current $\left(10^{-6} \mathrm{~A}\right)$ for PPy-based electrodes. (a) Normalized resistance changes upon sequential exposure to increasing concentrations of $\mathrm{NH}_{3}$ at different $\mathrm{pH}$ values. (b) Response and (c) recovery times of PPyNPs toward $0.1 \mathrm{ppm}$ of $\mathrm{NH}_{3}$ at different $\mathrm{pH}$ values. (d) Normalized resistance changes of $\mathrm{pH} 1$ based-electrode upon sequential exposure to decrease concentrations of $\mathrm{NH}_{3}$. (e) Response and (f) recovery times of $\mathrm{pH}$ 1 based-electrode toward $0.1 \mathrm{ppm}$ of $\mathrm{NH}_{3}$, depending on the direction of increase (black) and decrease (green) in concentration of $\mathrm{NH}_{3}$. 
(a)

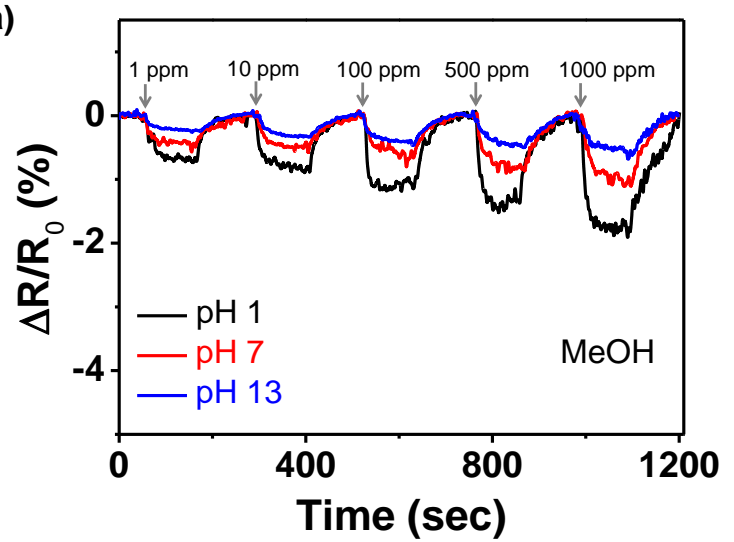

(b)

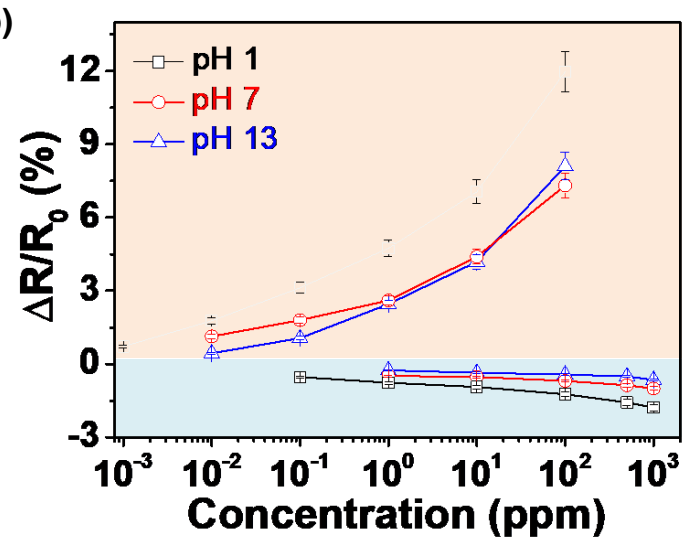

(c)

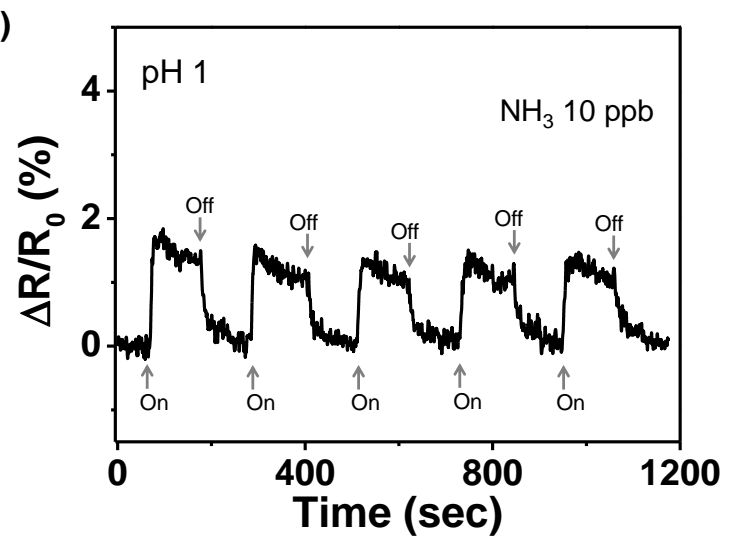

Figure 8. (a) Change in normalized resistance with different $\mathrm{MeOH}$ concentrations. (b) Calibration curves of PPyNPs as functions of $\mathrm{NH}_{3}$ and $\mathrm{MeOH}$ concentrations; black for $\mathrm{pH} 1$, red for $\mathrm{pH} 7$, and blue for $\mathrm{pH}$ 13. (c) Periodic exposure of PPyNPs treated at $\mathrm{pH} 1$ to $10 \mathrm{ppb}$ of $\mathrm{NH}_{3}$ gas.

The selectivity of the sensor electrodes is also important for practical applications. Figure 10 exhibits the variation in the normalized resistance of the electrode at $\mathrm{pH} 1$ upon exposure to various volatile gases at low concentrations ( $1 \mathrm{ppm}$ for $\mathrm{NH}_{3}$ and $\mathrm{MeOH} ; 100 \mathrm{ppm}$ for others). $\mathrm{NH}_{3}$ yields a signal change that is more than five times larger when compared to those of the other gases, although its concentration is lower than those of other gases. Therefore, $\mathrm{NH}_{3}$ can be distinguished from other gases based on the extent and direction of the resistance change. 


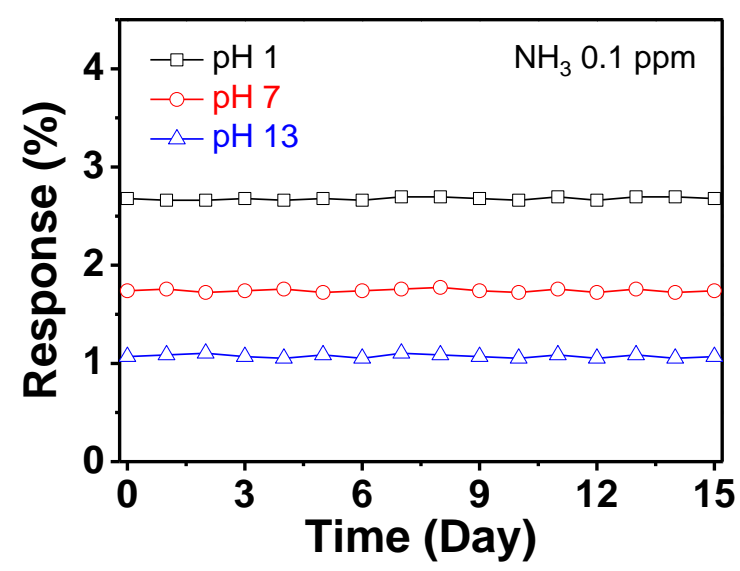

Figure 9. Normalized resistance changes of the electrodes with periodic exposure to $0.1 \mathrm{ppm}$ of $\mathrm{NH}_{3}$ for 15 days (black: $\mathrm{pH} 1$; red: $\mathrm{pH}$ 7; blue: $\mathrm{pH} 13$ ).

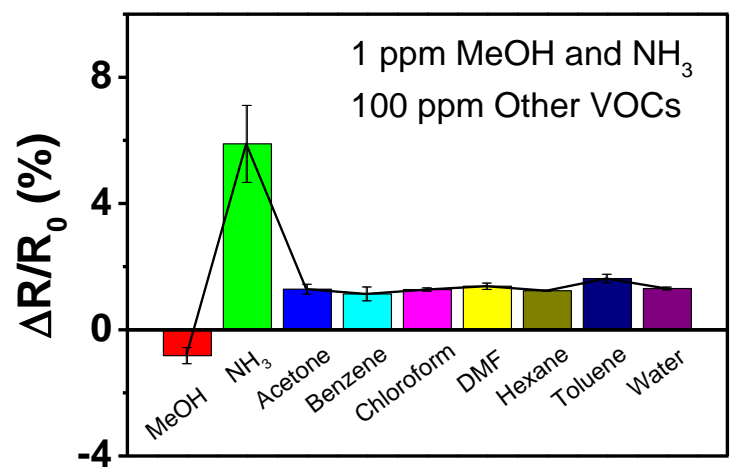

Figure 10. Sensing performance histogram of the PPyNPs treated at $\mathrm{pH} 1$ toward different oxidizing and reducing volatile gases. The concentrations of the gases are $1 \mathrm{ppm}$ for $\mathrm{NH}_{3}$ and $\mathrm{MeOH}$ and 100 ppm for other gases.

\section{Conclusions}

In summary, the chemical states of PPyNPs are investigated at various $\mathrm{pH}$ levels for application in hazardous-gas sensors. The structure of the polymer chain changes from the expanded to contracted state, with an increase in $\mathrm{pH}$. The nanoparticles at $\mathrm{pH} 1$ show higher electrical conductivity (128 S $\mathrm{cm}^{-1}$ ) and larger diameters than those at other $\mathrm{pH}$ levels, due to an increase in the amount of charge carriers (hole) in the chain structure and the osmotic effect of the counter ions. As an $\mathrm{NH}_{3}$ sensing transducer material, the nanoparticles at $\mathrm{pH} 1$ have short response and recovery times of less than $20 \mathrm{~s}$ and $50 \mathrm{~s}$, respectively, with high sensitivity (down to $1 \mathrm{ppb}$ ) at $25^{\circ} \mathrm{C}$. Thus, the performance of the conductive nanostructures is optimized through chemical-state control, and the proposed method can be effectively applied for the development of electrical devices and sensor systems.

Author Contributions: W.K. performed the experiments; S.C. conceived and designed the experiments; J.S.L. analyzed the data and wrote the paper. All authors have read and agreed to the published version of the manuscript.

Funding: This research received no external funding.

Acknowledgments: This work was supported by the National Research Foundation of Korea (NRF) grant funded by Korea government (NRF-2018R1C1B5041562).

Conflicts of Interest: The authors declare no conflict of interest. 


\section{References}

1. He, Y.; Li, D.; Gao, W.; Yin, H.; Chen, F.; Sun, Y. High-performance NO2 sensors based on spontaneously functionalized hexagonal boron nitride nanosheets via chemical exfoliation. Nanoscale 2019, 11, 21909-21916. [PubMed]

2. Jannat, A.; Haque, F.; Xu, K.; Zhou, C.; Zhang, B.Y.; Syed, N.; Mohiuddin, M.; Messalea, K.A.; Li, X.; Gras, S.L.; et al. Exciton-Driven Chemical Sensors Based on Excitation-Dependent Photoluminescent Two-Dimensional SnS. ACS Appl. Mater. Interfaces 2019, 11, 42462-42468. [PubMed]

3. Mi, H.-Y.; Liu, J.-L.; Guan, M.-M.; Liu, Q.-W.; Zhang, Z.-Q.; Feng, G.-D. Fluorescence chemical sensor for determining trace levels of nitroaromatic explosives in water based on conjugated polymer with guanidinium side groups. Talanta 2018, 187, 314-320. [PubMed]

4. Kim, W.; Lee, J.S.; Jang, J. Facile synthesis of size-controlled $\mathrm{Fe}_{2} \mathrm{O}_{3}$ nanoparticle-decorated carbon nanotubes for highly sensitive $\mathrm{H}_{2} \mathrm{~S}$ detection. RSC Adv. 2018, 8, 31874-31880.

5. Kim, S.G.; Jun, J.; Lee, J.S.; Jang, J. A highly sensitive wireless nitrogen dioxide gas sensor based on an organic conductive nanocomposite paste. J. Mater. Chem. A 2019, 7, 8451-8459.

6. Barbosa, M.S.; Suman, P.H.; Kim, J.J.; Tuller, H.L.; Orlandia, M.O. Investigation of electronic and chemical sensitization effects promoted by $\mathrm{Pt}$ and $\mathrm{Pd}$ nanoparticles on single-crystalline SnO nanobelt-based gas sensors. Sens. Actuator B-Chem. 2019, 301, 127055.

7. Kekkonen, J.; Finnilä, M.A.J.; Heikkilä, J.; Anttonen, V.; Nissinen, I. Chemical imaging of human teeth by a time-resolved Raman spectrometer based on a CMOS single-photon avalanche diode line sensor. Analyst 2019, 144, 6089-6097.

8. Si, H.; Pan, N.; Zhang, X.; Liao, J.; Rumyantseva, M.N.; Gaskov, A.M.; Lina, S. A real-time on-line photoelectrochemical sensor toward chemical oxygen demand determination based on field-effect transistor using an extended gate with $3 \mathrm{D} \mathrm{TiO}_{2}$ nanotube arrays. Sens. Actuator B-Chem. 2019, 289, 106-113.

9. Cho, S.-Y.; Jang, D.; Kang, H.; Koh, H.-J.; Choi, J.; Jung, H.-T. Ten Nanometer Scale $\mathrm{WO}_{3} / \mathrm{CuO}$ Heterojunction Nanochannel for an Ultrasensitive Chemical Sensor. Anal. Chem. 2019, 91, 6850-6858.

10. Broza, Y.Y.; Zhou, X.; Yuan, M.; Qu, D.; Zheng, Y.; Vishinkin, R.; Khatib, M.; Wu, W.; Haick, H. Disease Detection with Molecular Biomarkers: From Chemistry of Body Fluids to Nature-Inspired Chemical Sensors. Chem. Rev. 2019, 119, 11761-11817.

11. Piro, B.; Mattana, G.; Noël, V. Recent Advances in Skin Chemical Sensors. Sensors 2019, 19, 4376.

12. Jang, J. Conducting Polymer Nanomaterials and Their Applications. Adv. Polym. Sci. 2006, 199, 189-259.

13. Cho, S.; Lee, J.S.; Joo, H. Recent Developments of the Solution-Processable and Highly Conductive Polyaniline Composites for Optical and Electrochemical Applications. Polymers 2019, 11, 1965.

14. Moon, J.-M.; Thapliyal, N.; Hussain, K.K.; Goyal, R.N.; Shim, Y.-B. Conducting polymer-based electrochemical biosensors for neurotransmitters: A review. Biosens. Bioelectron. 2018, 102, 540-552.

15. Wang, G.; Morrin, A.; Li, M.; Liu, N.; Luo, X. Nanomaterial-doped conducting polymers for electrochemical sensors and biosensors. J. Mater. Chem. B 2018, 6, 4173-4190.

16. Park, S.J.; Park, C.S.; Yoon, H. Chemo-Electrical Gas Sensors Based on Conducting Polymer Hybrids. Polymers 2017, 9, 155.

17. Li, S.; Liu, A.; Yang, Z.; He, J.; Wang, J.; Liu, F.; Lu, H.; Yan, X.; Sun, P.; Liang, X.; et al. Room temperature gas sensor based on tin dioxide@ polyaniline nanocomposite assembled on flexible substrate: Ppb-level detection of $\mathrm{NH}_{3}$. Sens. Actuator B-Chem. 2019, 299, 126970.

18. Bhadra, J.; Popelka, A.; Abdulkareem, A.; Ahmad, Z.; Touati, F.; Al-Thani, N. Fabrication of polyaniline-graphene/polystyrene nanocomposites for flexible gas sensors. RSC Adv. 2019, 9, 12496-12506.

19. Hassan, G.; Sajid, M.; Choi, C. Highly Sensitive and Full Range Detectable Humidity Sensor using PEDOT: PSS, Methyl Red and Graphene Oxide Materials. Sci. Rep. 2019, 9, 15227.

20. Zhang, Q.; Wang, X.; Fu, J.; Liu, R.; He, H.; Ma, J.; Yu, M.; Ramakrishna, S.; Long, Y. Electrospinning of Ultrafine Conducting Polymer Composite Nanofibers with Diameter Less than $70 \mathrm{~nm}$ as High Sensitive Gas Sensor. Materials 2018, 11, 1744.

21. Cho, K.H.; Shin, D.H.; Oh, J.; An, J.H.; Lee, J.S.; Jang, J. Multidimensional Conductive Nanofilm-Based Flexible Aptasensor for Ultrasensitive and Selective HBsAg Detection. ACS Appl. Mater. Interfaces 2018, 10, 28412-28419. [PubMed] 
22. Lee, J.S.; Kim, S.G.; Jun, J.; Shin, D.H.; Jang, J. Aptamer-Functionalized Multidimensional Conducting-Polymer Nanoparticles for an Ultrasensitive and Selective Field-Effect-Transistor Endocrine-Disruptor Sensors. Adv. Funct. Mater. 2014, 24, 6145-6153.

23. Lee, J.S.; Kim, W.; Cho, S.; Jun, J.; Cho, K.H.; Jang, J. Multidimensional hybrid conductive nanoplate-based aptasensor for platelet-derived growth factor detection. J. Mater. Chem. B 2016, 4, 4447-4454.

24. Pan, L.; Yu, G.; Zhai, D.; Lee, H.R.; Zhao, W.; Liu, N.; Wang, H.; Tee, B.C.-K.; Shi, Y.; Cui, Y.; et al. Hierarchical nanostructured conducting polymer hydrogel with high electrochemical activity. Proc. Natl. Acad. Sci. USA 2012, 109, 9287-9292.

25. Shen, L.; Huang, X. Tuning the morphologies and electrical properties of azobenzene- $4,4^{\prime}$-dicarboxylate-doped polypyrrole via ultraviolet light irradiation and medium $\mathrm{pH}$ alteration. Polymers 2019, 176, 188-195.

26. Santino, L.M.; Diao, Y.; Yang, H.; Lu, Y.; Wang, H.; Hwang, E.; D'Arcy, J.M. Vapor/liquid polymerization of ultraporous transparent and capacitive polypyrrole nanonets. Nanoscale 2019, 11, 12358-12369.

27. Hea, W.; Zhao, G.; Sun, P.; Hou, P.; Zhu, L.; Wang, T.; Lib, L.; Xu, X.; Zhai, T. Construction of Longan-like hybrid structures by anchoring nickel hydroxide on yolk-shell polypyrrole for asymmetric supercapacitors. Nano Energy 2019, 56, 207-215.

28. Tang, X.; Raskin, J.-P.; Kryvutsa, N.; Hermans, S.; Slobodian, O.; Nazarovc, A.N.; Debliquy, M. An ammonia sensor composed of polypyrrole synthesized on reduced graphene oxide by electropolymerization. Sens. Actuator B-Chem. 2020, 305, 127423.

29. Domínguez-Renedo, O.; Navarro-Cuñado, A.M.; Arnáiz-Lozano, V.; Alonso-Lomillo, M.A. Molecularly imprinted polypyrrole based electrochemical sensor for selective determination of 4-ethylphenol. Talanta 2020, 207, 120351.

30. Jun, J.; Oh, J.; Shin, D.H.; Kim, S.G.; Lee, J.S.; Kim, W.; Jang, J. Wireless, Room Temperature Volatile Organic Compound Sensor Based on Polypyrrole Nanoparticle Immobilized Ultrahigh Frequency Radio Frequency Identification Tag. ACS Appl. Mater. Interfaces 2016, 8, 33139-33147.

31. Camurlu, P. Polypyrrole derivatives for electrochromic applications. RSC Adv. 2014, 4, 55832-55845.

32. Patil, A.O.; Heeger, A.J.; Wudl, F. Optical Properties of Conducting Polymers. Chem. Rev. 1988, 88, $183-200$.

33. Arjomandi, J.; Shah, A.-u.-H.A.; Bilal, S.; Hoang, H.V.; Holze, R. In situ Raman and UV-vis spectroscopic studies of polypyrrole and poly(pyrrole-2,6-dimethyl- $\beta$-cyclodextrin). Spectroc. Acta Pt. A-Molec. Biomolec. Spectr. 2011, 78, 1-6.

34. Santos, M.J.L.; Brolo, A.G.; Girotto, E.M. Study of polaron and bipolaron states in polypyrrole by in situ Raman spectroelectrochemistry. Electrochim. Acta 2007, 52, 6141-6145.

35. Nguyen Thi Le, H.; Bernard, M.C.; Garcia-Renaud, B.; Deslouis, C. Raman spectroscopy analysis of polypyrrole films as protective coatings on iron. Synth. Met. 2004, 140, 287-293.

36. Carrasco, P.M.; Cortazar, M.; Ochoteco, E.; Calahorra, E.; Pomposo, J.A. Comparison of surface and bulk doping levels in chemical polypyrroles of low, medium and high conductivity. Surf. Interface Anal. 2006, 39, $26-32$.

37. Joo, J.; Lee, J.K.; Lee, S.Y.; Jang, K.S.; Oh, E.J.; Epstein, A.J. Physical Characterization of Electrochemically and Chemically Synthesized Polypyrroles. Macromolecules 2000, 33, 5131-5136.

38. Li, Y.; He, G. Effect of preparation conditions on the two doping structures of polypyrrole. Synth. Met. 1998, 94, 127-129.

39. Cheah, K.; Forsyth, M.; Truong, V.-T. Ordering and stability in conducting polypyrrole. Synth. Met. 1998, 94, 215-219.

(C) 2020 by the authors. Licensee MDPI, Basel, Switzerland. This article is an open access article distributed under the terms and conditions of the Creative Commons Attribution (CC BY) license (http://creativecommons.org/licenses/by/4.0/). 